\title{
Effect of Ingested Culture of Propionibacterium freudenreichii ET-3 on Fecal Microflora and Stool Frequency in Healthy Females
}

\author{
Kenichi Hojo, ${ }^{1 *}$ Nobuo Yoda, ${ }^{1}$ Hiroshi Tsuchita, ${ }^{2}$ Toshihiro Ohtsu, ${ }^{3}$ Keisuke Sekı, ${ }^{3}$ Naoki Taketomo, ${ }^{1}$ \\ Tsutomu Murayama ${ }^{4}$ and Hisakazu lino ${ }^{5}$ \\ ${ }^{1}$ Food Functionality Research Institute, Meiji Dairies Corporation, 540 Naruda, Odawara 250-0862, Japan \\ ${ }^{2}$ Nutrition Science Institute, Meiji Dairies Corporation, 1-21-3 Sakae-cho, Higashimurayama 189-8530, Japan \\ ${ }^{3}$ Nutritional Foods Development Department, Meiji Dairies Corporation, 1-26-11 Midori, Sumida-ku, Tokyo 130-8502, Japan \\ ${ }^{4}$ Department of Home Economics, Odawara Women's Junior College, 4-5-1 Shiroyama, Odawara 250-0045, Japan \\ ${ }^{5}$ Science for Living System, Graduate School of Showa Women's University, 1-7 Taishido, Setagaya-ku, Tokyo 154-8533, Japan \\ Received September 18, 2001; Accepted for publication, January 8, 2002
}

\begin{abstract}
The effect of the ingested culture of Propionibacterium freudenreichii ET-3 that produced bifidogenic growth stimulator (BGS) on stool frequency and quantity was investigated in 41 young healthy women in a randomized double-blind crossover study. The fecal samples of 7 of these 41 subjects were analyzed to study the modification of intestinal microflora by BGS. The results were compared with the effects of an ingested placebo tablet. The ratio of bifidobacteria population $(\%)$ to total bacteria population during the BGS-tablet intake period was significantly increased $(p<0.05)$ in comparison with that during the placebo tablet intake period. There were no significant differences in fecal pH and water content during the experimental periods. However, the putrefactive metabolites, namely, fecal indole and skatole concentration, of the BGS-tablet intake period significantly decreased $(p<0.05)$ in comparison with those of the placebo tablet period. The stool quantity of the BGS-tablet intake period was slightly higher $(p=0.083)$ than that of the nonintake period. Moreover, the stool frequency of the BGS-tablet intake period chosen from those originally with lower frequency ( $\leqq 4$ times/week) was significantly higher $(p<0.05)$ than that of the placebo tablet intake period. These results show that the ingestion of $P$. freudenreichii ET-3 culture increases the number of defecations of constipated females, indicating an improvement of intestinal microflora.
\end{abstract}

Key words: Bifidobacterium; Prebiotics; BGS; putrefactive products; defecation

\section{INTRODUCTION}

There is a large variety of bacterial genera, species, and strains in the human intestine; these bacteria are either beneficial (e.g., Bifidobacterium and Lactobacillus) or harmful (e.g., Clostridium, Salmonella and Staphylococcus) to the host's health. Bifidobacteria are a major bacterial group in the normal intestinal microflora (4) and may play an important role in the ecophysiology of the microflora (7). As the effects of these bacteria have been revealed, there is currently much interest in the studies of prebiotics that stimulate the growth of beneficial bacteria in the intestine. A prebiotic is defined as "a nondigestible food ingredient that beneficially affects the host by selectively stimulating the growth and/or activity of one or a limited number of bacteria in the colon" (6). In general, the prebiotics developed to date are nondigestible oligosaccharides.

\footnotetext{
*Corresponding author. Mailing address: Food Functionality Research Institute, Meiji Dairies Corporation, 540 Naruda, Odawara 250-0862, Japan. Phone: +81-465-37-3661. Fax: +81-465-36-2776.
}

Kaneko et al. (9) found a novel bifidogenic growth stimulator (BGS) produced by Propionibacterium freudenreichii, which is used extensively in the dairy industry as a starter culture for the manufacture of Swiss-type cheese. The BGS has a specific effect on the bifidobacterial growth at an extremely low concentration (9). The BGS was produced both in cell free supernatant and in the nonpolar solvent extraction of the cell $(9,13)$. In regard to prebiotic effects, it was reported that the bifidobacterial count and its ratio to total bacteria were increased when sterilized $P$. freudenreichii culture was added to anaerobic continuous culture system simulating the bacterial ecosystem of human intestine $(8,10)$. In in vitro models, moreover, harmful bacteria such as Clostridium perfringens and enterobacteriaceae were greatly decreased by the addition of sterilized $P$. freudenreichii culture (10). However, to understand the potential value of the BGS and to progress in the development of the new prebiotics, an in vivo study is required.

The objective of the present study is to conclude whether the BGS acts as a useful bifidogenic factor in 
the human intestine. We studied the efficacy of the tablet containing $P$. freudenreichii culture on the bacterial composition of feces, fecal putrefactive products, and stool frequency and quantity in this double-blind, placebo-controlled crossover study.

\section{MATERIALS AND METHODS}

Bacteria. P. freudenreichii ET-3 used in this study was isolated from a Swiss-type cheese, and Bifidobacterium longum 6001 used as test microorganism for BGS activity assay is stocked in the Food Functionality Research Institute of Meiji Dairies Co., Odawara, Japan.

Assay of BGS activity. BGS activity was determined by an agar diffusion plate assay according to the method of Satomi et al. (17). A sterile paper disk $6 \mathrm{~mm}$ in diameter (Toyo Roshi Co., Tokyo, Japan) with $10 \mu \mathrm{l}$ of the sample was placed on the surface of the plate containing $B$. longum 6001 and incubated anaerobically at $37^{\circ} \mathrm{C}$ for $15 \mathrm{hr}$. The diameter of the growth zone was measured directly after incubation. To determine the BGS activity quantitatively, 1-hydroxy-2-naphthoic acid solution was used as a standard. One unit of BGS activity was defined as the amount of BGS, which showed a growth diameter equal to that produced by 1 $\mu \mathrm{g} / \mathrm{ml}$ of the standard solution.

Sample preparation. The test materials were administered in tablet form. The composition of the tablets is shown in Table 1. P. freudenreichii ET-3 was grown at $35^{\circ} \mathrm{C}$ for $72 \mathrm{hr}$ in $10 \%$ (wt/wt) milk whey medium, which was digested by Protease Amano A (Amano Pharmaceutical Co., Tokyo, Japan). During cultivation, the $\mathrm{pH}$ of the medium was adjusted to 6.0 , using $8 \mathrm{~N} \mathrm{~K}_{2} \mathrm{CO}_{3}$ as a neutralizer. The culture was lyophilized and used for preparation of the foodstuff. Each subject was given 3 tablets $(1.5 \mathrm{~g})$ per day orally, which contained $24.8 \mathrm{U}$ of BGS activity. Three tablets contained $0.03 \mathrm{~g}$ of protein, $0.04 \mathrm{~g}$ of lipid, $1.3 \mathrm{~g}$ of carbohydrate, and $3.0 \mathrm{mg}$ of sodium. Placebo tablets were prepared with lyophilized whey medium instead of $P$. freudenreichii ET-3 culture.

Subjects. The study protocol was approved by the Ethical Committee of Showa Women's University and Meiji Dairies Co. Sixty-three healthy female university students aged $18-23$ years (mean age $20.8 \pm 3.8$, weight $51.2 \pm 6.5 \mathrm{~kg}$, and height $157.7 \pm 6.0 \mathrm{~cm}$ ) were recruited into the study after their informed consents were received. Excluding the volunteers that ingested medicine such as antibiotics and dropped out because of neglect in recording stool condition, 41 volunteers (mean age $21.0 \pm 4.3$, weight $51.6 \pm 7.4 \mathrm{~kg}$, and height
Table 1. Composition [wt/wt (\%)] of the test tablets.

\begin{tabular}{lcc}
\hline & Placebo tablets & BGS tablets \\
\hline Fermented medium* & - & 20.0 \\
Medium & 20.0 & - \\
Glucose & 75.5 & 75.5 \\
Emulsifier & 3.0 & 3.0 \\
Flavors & 1.5 & 1.5 \\
\hline Total & 100.0 & 100.0 \\
\hline
\end{tabular}

*Lyophilized milk whey medium fermented by Propioni-

bacterium freudenreichii.

$157.6 \pm 5.5 \mathrm{~cm}$ ) took part in this study. Each subject was assigned to one of two classes on the basis of the stool frequency observed for the control period (normal: $>4$ times/week, lower: $\leqq 4$ times/week). The fecal samples of 7 subjects (mean age $19.1 \pm 0.8$, weight $51.5 \pm 5.8 \mathrm{~kg}$, and height $158.0 \pm 5.4 \mathrm{~cm}$ ) of all subjects were analyzed to evaluate the effect of BGS on the intestinal microflora.

Study design. In this double-blind, placebo-controlled crossover study, the experimental period comprised four two-week periods. The first of the experimental period was the control period. During the following 14 days, all subjects were administrated test tablets (3 per day) containing $P$. freudenreichii culture or a placebo after a meal, and neither the content of the tablets nor the group assignments were known to any of the subjects. The other test tablets were ingested after an interval of a 14-day nonintake period. The subjects followed their normal diet, but they were asked to consume no products containing probiotics or prebiotics and to limit the intake of alcoholic drinks. During the experimental period, the subjects provided the records of stool frequencies and quantities and general health conditions every day, as described below. Stool samples for the bacteriological and chemical analyses were collected on a random day between days 12 and 14 of each experimental period.

Bacteriologic analysis. An enumeration of bacterial populations in the feces was carried out under methods developed by Mitsuoka (11). The samples obtained from each subject were introduced into an anaerobic chamber $\left(\mathrm{N}_{2}, \mathrm{H}_{2}, \mathrm{CO}_{2}: 80 \%, 10 \%, 10 \%\right.$ atmosphere). Then $1 \mathrm{~g}$ of the sample was suspended in $9 \mathrm{ml}$ of trypticase soy broth without dextrose (BBL), supplemented with $0.084 \%$ (w/v) $\mathrm{Na}_{2} \mathrm{CO}_{3}, 0.05 \%$ (w/v) agar, and $0.05 \%(\mathrm{w} / \mathrm{v})$ L-cysteine hydrochloride. Serial tenfold dilutions from $10^{-1}$ to $10^{-8}$ were prepared of the same medium. Aliquots $(0.1 \mathrm{ml})$ of each dilution were plated onto nonselective media or various selective media. After incubation, fecal bacteria were identified 
by colony and cell morphology, Gram-reactions, aerobic and anaerobic growth, and biochemical tests. The number of total bacteria and each genus was expressed in terms of colony forming unit per gram of feces.

Measurements of fecal $\mathrm{pH}$, water, and ammonia. The fecal $\mathrm{pH}$ was determined by using a glass $\mathrm{pH}$ electrode. The water content of feces was calculated by subtracting the weight after drying at $98^{\circ} \mathrm{C}$ for $18 \mathrm{hr}$ from the weight of the wet feces. Ammonia was measured spectrophotometrically with a commercially available kit (Boehringer GmbH, Mannheim, Germany).

Determination of putrefactive products in feces. The putrefactive products in feces were measured by gas chromatography. The fecal samples were diluted tenfold with distilled water. Ten grams of fecal slurry was transferred into a centrifuge tube, and the $\mathrm{pH}$ of samples was adjusted to 7.0 by $2 \mathrm{~N} \mathrm{NaOH}$ after the addition of 4 $\mathrm{g}$ of sodium chloride. The putrefactive products were extracted with $30 \mathrm{ml}$ of diethyl ether by shaking. After centrifugation at $3000 \mathrm{rpm}$ for $5 \mathrm{~min}$, the supernatant was collected. The extraction was carried out twice, and both extracts were combined. One $\mu$ l of the concentrated ether layer was injected onto a capillary column (TC-1, $30 \mathrm{~m} \times 0.25 \mathrm{~mm}$, GL Sciences Inc., Tokyo, Japan) in a Hewlett-Packard Model 5890 Gas Chromatograph. The column oven was programmed to increase to $240^{\circ} \mathrm{C}$, from $60^{\circ} \mathrm{C}$, at $5^{\circ} \mathrm{C}$ per min, and the temperature of the injection port and flame ionization detector was $210^{\circ} \mathrm{C}$.

Assessment of questionnaire. The items of the quantity of stools were converted to numerical data as follows. The stool quantity was estimated by participants as the number of cakes of soap it was equivalent to. They also recorded their intake of any supplements for constipation, such as fermented milk or dietary fiber, and the intake of medicine, such as antibiotics or other drugs. All data were collected and reviewed by the investigators responsible for this study.

Statistical analysis. A Wilcoxon signed rank-sum test was used for the statistical analysis of the data. A probability of less than 0.05 was taken to indicate statistical significance.

\section{RESULTS}

\section{Composition of Intestinal Microflora}

Several groups of intestinal bacteria were enumerated to determine the microbial composition of feces (Table 2). The bifidobacteria/total bacteria ratio during the BGS tablet intake period was significantly increased $(p<0.05)$ in comparison with those during the placebo tablet intake and control period. The number of bacteroidaceae in feces of the BGS tablet intake period was

Table 2. Mean bacterial counts ( $\log _{10} / g$ of feces) in fecal samples of 7 volunteers.

\begin{tabular}{lcccc}
\hline & Control & Placebo tablets & Nonintake & BGS tablets \\
\hline Total bacteria & $10.71 \pm 0.45$ & $10.69 \pm 0.29$ & $10.68 \pm 0.25$ & $10.67 \pm 0.16$ \\
Bacteroidaceae & $10.33 \pm 0.53$ & $10.34 \pm 0.34$ & $10.27 \pm 0.33$ & $10.04 \pm 0.51$ \\
Bifidobacteria & $(100)^{*}$ & $(100)$ & $(100)$ & $(100)$ \\
& $10.16 \pm 0.56$ & $10.16 \pm 0.28$ & $10.11 \pm 0.38$ & $10.26 \pm 0.21$ \\
Eubacteria & $(100)$ & $(100)$ & $(100)$ & $(100)$ \\
& $9.95 \pm 0.28$ & $9.89 \pm 0.38$ & $9.83 \pm 0.28$ & $9.92 \pm 0.27$ \\
Clostridia & $(100)$ & $(100)$ & $(100)$ & $(100)$ \\
& $8.96 \pm 0.38$ & $9.01 \pm 0.91$ & $9.12 \pm 0.41$ & $8.91 \pm 0.66$ \\
Anaerobic cocci & $(85.71)$ & $(71.43)$ & $(100)$ & $(85.71)$ \\
& $9.06 \pm 0.71$ & $9.03 \pm 0.43$ & $9.42 \pm 0.29$ & $9.26 \pm 0.32$ \\
Lactobacilli & $(100)$ & $(85.71)$ & $(85.71)$ & $(85.71)$ \\
& $5.40 \pm 2.06$ & $5.43 \pm 2.08$ & $5.31 \pm 1.89$ & $5.40 \pm 1.78$ \\
Lecithinase positive bacteria & $(71.43)$ & $(57.14)$ & $(71.43)$ & $(42.86)$ \\
Enterobacteriaceae & $3.85 \pm 0.74$ & $3.95 \pm 0.56$ & $4.53 \pm 1.30$ & $4.30 \pm 1.07$ \\
Streptococci & $(85.71)$ & $(85.71)$ & $(71.43)$ & $(57.14)$ \\
Bifidobacteria $(\%)^{\dagger}$ & $7.27 \pm 0.97$ & $6.55 \pm 1.11$ & $7.64 \pm 0.47$ & $7.90 \pm 0.53$ \\
& $(100)$ & $(100)$ & $(85.71)$ & $(85.71)$
\end{tabular}

Mean values in the same row that are significantly different at $p<0.05$ are indicated with different letter superscripts.

*Frequency of occurrence.

${ }^{\dagger}$ Bifidobacteria population (\%) to total fecal bacteria. 
Table 3. Changes in putrefactive products of 7 volunteers during experimental periods.

\begin{tabular}{lcccc}
\hline & Control & Placebo tablets & Nonintake & BGS tablets \\
\hline Phenol $(\mu \mathrm{g} / \mathrm{g}$ wet feces $)$ & $9.10 \pm 8.34$ & $4.99 \pm 7.01$ & $3.64 \pm 3.23$ & $4.63 \pm 4.41$ \\
$p$-Cresol $(\mu \mathrm{g} / \mathrm{g}$ wet feces $)$ & $107.38 \pm 83.03$ & $47.10 \pm 11.09$ & $84.11 \pm 80.70$ & $52.05 \pm 40.09$ \\
Indole $(\mu \mathrm{g} / \mathrm{g}$ wet feces $)$ & $66.31 \pm 59.94^{\mathrm{a}}$ & $39.60 \pm 11.23^{\mathrm{a}}$ & $47.64 \pm 28.36^{\mathrm{a}}$ & $25.81 \pm 16.32^{\mathrm{b}}$ \\
Skatole $(\mu \mathrm{g} / \mathrm{g}$ wet feces $)$ & $18.56 \pm 17.88^{\mathrm{ab}}$ & $27.06 \pm 2.84^{\mathrm{a}}$ & $16.23 \pm 12.31^{\mathrm{ab}}$ & $7.35 \pm 9.56^{\mathrm{b}}$ \\
Ammonia $(\mu \mathrm{g} / \mathrm{g}$ wet feces $)$ & $511.93 \pm 334.95$ & $508.36 \pm 333.76$ & $437.81 \pm 224.48$ & $312.70 \pm 104.18$ \\
& & & & \\
Water $(\%)$ & $68.46 \pm 8.61$ & $76.09 \pm 3.48$ & $69.69 \pm 8.43$ & $74.29 \pm 7.68$ \\
$\mathrm{pH}$ & $6.47 \pm 0.63$ & $6.77 \pm 0.35$ & $6.62 \pm 0.62$ & $6.56 \pm 0.41$ \\
\hline
\end{tabular}

Values are of the mean \pm SD.

Mean values in the same row that are significantly different at $p<0.05$ are indicated with different letter superscripts.

Table 4. Effect of ingested tablets containing Propionibacterium culture on frequency of evacuation and stool quantity.

\begin{tabular}{cllcll}
\hline & & Control & Placebo tablets & Nonintake & BGS tablets \\
\hline $\begin{array}{c}\text { Lower }(n=19) \\
\leqq 4 \text { times/week }\end{array}$ & Frequency & $3.03 \pm 0.82^{\mathrm{a}}$ & $3.42 \pm 1.72^{\mathrm{a}}$ & $3.45 \pm 1.12^{\mathrm{ab}}$ & $3.97 \pm 2.41^{\mathrm{b}}$ \\
& Quantity & $5.31 \pm 2.49$ & $6.12 \pm 2.64$ & $5.63 \pm 2.82$ & $6.91 \pm 4.14$ \\
$\begin{array}{c}\text { Normal }(n=22) \\
>4 \text { times/week }\end{array}$ & Frequency & $6.36 \pm 2.01$ & $6.70 \pm 2.70$ & $6.09 \pm 2.75$ & $6.34 \pm 2.58$ \\
& Quantity & $9.43 \pm 4.52$ & $10.92 \pm 5.80$ & $9.32 \pm 5.47$ & $9.47 \pm 5.17$ \\
All subjects $(n=41)$ & & & & \\
& Frequency & $4.82 \pm 2.30$ & $5.18 \pm 2.81$ & $4.87 \pm 2.52$ & $5.24 \pm 2.75$ \\
& Quantity & $7.52 \pm 4.22^{\mathrm{a}}$ & $8.70 \pm 5.16^{\mathrm{b}}$ & $7.61 \pm 4.77^{\mathrm{ab}}$ & $8.28 \pm 4.84^{\mathrm{ab}}$ \\
\hline
\end{tabular}

Values are of the mean \pm SD.

Mean values in the same row that are significantly different at $p<0.05$ are indicated with different letter superscripts.

lower $(p=0.091)$ than those of the placebo intake period, though there were no significant changes in other bacterial groups.

\section{Fecal pH, Water, and Metabolite Concentration}

The results in Table 3 show the change of the putrefactive products produced by the degradation of aromatic amino acids by intestinal bacteria during the experimental period. Compared with other experimental periods, fecal indole concentration during the intake of BGS tablets was significantly lowest $(p<0.05)$. During the BGS tablet intake period, fecal skatole concentration also decreased $(p<0.01, p=0.063)$ in comparison with those of the placebo tablet intake and nonintake periods. There were no significant differences in fecal $\mathrm{pH}$ or water content.

\section{Questionnaire Result}

Two classes of subjects were separated according to their original stool frequency on the basis of data during the control period. The mean values of stool frequency are shown in Table 4. The stool frequency of the subjects in the lower frequency class ( $\leqq 4$ times/ week) during BGS tablet intake was significantly higher $(p<0.05)$ than for those with control and placebo tablet intake. The change in stool quantity in the lower frequency class seemed to be consistent with the change in stool frequency. The stool quantity of the BGS tablet intake period was greater than that of the control period $(p=0.088)$ and nonintake period $(p=0.061)$.

For subjects of the normal class ( $>4$ times/week), there was no significant difference in stool frequency during the experimental period. The stool quantity of the BGS tablet intake period for all subjects was slightly higher $(p=0.083)$ than that of the nonintake period, and during placebo tablet intake period it significantly increased $(p<0.05)$ compared with that during the control period.

\section{DISCUSSION}

It is believed that certain components in human breast milk such as high lactose, oligosaccharides, $\mathrm{N}$-acetylglucosamine-containing saccharides, glycoproteins, and glucopeptides are growth factors for bifidobacteria (12, 16). Indigestible carbohydrates, pantethine, lactulose, $\kappa$-casein, trypsin digest, and products of aminocarbonyl 
reaction also enhance the growth of bifidobacteria ( 2 , $5,15,16)$. Kaneko et al. (9) found that $P$. freudenreichii used traditionally as a Swiss-type cheese starter produced bifidogenic growth stimulator. In the culture of human feces, $P$. freudenreichii culture stimulated specifically the growth of bifidobacteria in the presence of several intestinal bacteria $(8,10)$. This study, using human subjects, investigated whether the bacterial flora in the intestine and certain indexes of intestinal metabolism could be influenced by the consumption of BGS tablets made from $P$. freudenreichii culture.

The analysis of bacterial counts in feces revealed that bifidobacteria/ total bacteria ratio significantly increased after BGS tablet intake, but not after placebo tablet intake, and bacteroidaceae during a BGS tablet intake period was lower than that during a placebo intake period. By means of an anaerobic continuous culture system simulating the gut bacterial ecosystem, $P$. freudenreichii culture also enhanced bifidobacterial percentage when a substrate was simultaneously added into that system, though bifidobacterial percentage decreased without $P$. freudenreichii culture (8). It was suggested that the substrate was utilized by gut bacteria other than bifidobacteria, but that the $P$. freudenreichii culture with BGS most likely enhanced prior utilization of the substrate by bifidobacteria (8). In this study, the growth stimulation of bifidobacteria may affect the decrease in bacteroidaceae during the BGS tablet intake period because bifidobacteria overwhelm their opponents in competition for the utilization of limited substrate in the intestine.

Although we found no significant differences between the fecal $\mathrm{pH}$ and ammonia concentration during the placebo and the BGS tablet intake periods, both indole and skatole ( $\beta$-methylindole) concentrations during the BGS tablet intake period were significantly decreased in comparison with those during the placebo tablet intake period. The fecal indolic and phenolic compounds are associated with a variety of disease states in humans and animals. These compounds have been implicated in cancer, but they seem to act as promoters of the disease instead of being directly carcinogenic by themselves $(3,18)$. Because the indolic compounds are produced from tryptophan by intestinal bacteria, the consumption of BGS tablets would be helpful in modifying the microbial metabolism in humans.

According to the experiment design, the investigation of stool frequency and quantity by questionnaire was continued for 8 weeks, and none of the subjects mentioned problems such as abdominal pain and flatulence. Division of the subjects into two classes on the basis of stool frequency in the control period afforded interesting results upon comparison of the data for each experimental period. The stool frequency during the BGS tablet intake period for subjects in the lower-frequency class showed a significant increase as compared with those in the control period and the placebo tablet intake period. However, for subjects in the normal class, the inherent frequency was not affected by BGS tablet intake. These results indicate that the ingestion of BGS tablets increases the number of defecations of constipated volunteers to improve the balance of colonic bacteria toward a potentially healthier microflora.

It is interesting that the effective bifidogenic factor was produced by microorganisms used for foods. We identified that 1,4-dihydroxy-2-naphthoic acid (DHNA), which is an intermediate of vitamin $\mathrm{K}_{2}$ (menaquinone) biosynthesis by microorganisms (1), is one of the principal ingredients in the BGS action of $P$. freudenreichii culture (unpublished data). Three BGS tablets per day given in this study were equivalent to $6.6 \mu \mathrm{g}$ of DHNA per day (unpublished data). Newman et al. (14) reported that menaquinone, or DHNA, is used in the electron transport chain of a certain microorganism as a shuttle to electron acceptors. It is not clear what role menaquinone plays in anaerobic bacteria. Moreover, bifidobacteria do not produce menaquinone or DHNA. We hypothesize that bifidobacteria supplement its intake of DHNA, a quinone structure compound and a naphthoic acid that may be necessary for the growth of bifidobacteria, with its intake of the leftover quinones synthesized by quinone-producing bacteria in the intestine. Research focusing on the kinetics of DHNA in the digestive tract and mechanism employing DHNA in bifidobacteria is now in progress.

Acknowledgments. We thank Ms. Hiroko Takamatsu, Ms. Chinami Mizoguchi, Ms. Saori Kurama, Ms. Mika Shibasaki, and Mr. Kakuhei Isawa for their helpful assistance and discussion.

\section{REFERENCES}

(1) Bentley R, Meganathan R. 1982. Biosynthesis of vitamin K (menaquinone) in bacteria. Microbiol Rev 46: 241-280.

(2) Djouzi Z, Andrieux C. 1997 Compared effects of three oligosaccharides on metabolism of intestinal microflora in rats inoculated with a human fecal flora. Br J Nutr 78: 313324.

(3) Dunning WF, Curtis MR, Maun ME. 1950. The effect of added dietary tryptophane on the occurrence of 2-acetylaminofluorene-induced liver and bladder cancer in rats. Cancer Res 10: 454-459.

(4) Finegold SM, Sutter VL, Mathisen GE. 1983. Normal in- 
digenous intestinal flora. In Human Intsetinal Microflora in Health and Disease, Hentges DJ (ed), Academic Press, London, p. 265-284.

(5) Furukawa H, Matsuo H, Deguchi T, Shiga A, Samejima H. 1968. Growth-promoting factors for Lactobacillus bifidus var. pennsylvanicus produced by the amino-carbonyl reaction of L-lysine and D-glucose. Agric Biol Chem 32: 617632.

(6) Gibson GR, Roberfroid MB. 1995. Dietary modulation of the human colonic microbiota-Introducing the concept of prebiotics. J Nutr 125: 1401-1412.

(7) Gibson GR, Wang X. 1994. Regulatory effects of bifidobacteria on the growth of other colonic bacteria. $\mathrm{J}$ Appl Bacteriol 77: 412-420.

(8) Kaneko T. 1999. A novel bifidogenic growth stimulator produced by Propionibacterium freudenreichii. Bioscience Microflora 18: 73-80.

(9) Kaneko T, Mori H, Iwata M, Meguro S. 1994. Growth stimulator for bifidobacteria produced by Propionibacterium freudenreichii and several intestinal bacteria. J Dairy Sci 77: 393-404.

(10) Kaneko T, Noda K. 1996. Bifidogenic growth stimulator produced by propionic acid bacteria. Jpn J Dairy Food Sci 45: 83-91 (in Japanese).

(11) Mitsuoka T. 1980. A Color Atlas of Anaerobic Bacteria,
Sobunsha, Tokyo, p. 13-92, 319-327 (in Japanese).

(12) Modler HW, Mckellar RC, Yaguchi M. 1990. Bifidobacteria and bifidogenic factors. Can Inst Food Sci Technol J 23: $29-41$.

(13) Mori H, Sato Y, Taketomo N, Kamiyama T, Yoshiyama Y, Meguro S, Sato H, Kaneko T. 1997. Isolation and structural identification of bifidogenic growth stimulator produced by Propionibacterium freudenreichii. J Dairy Sci 80: 1959-1964.

(14) Newman DK, Kolter R. 2000. A role for excreted quinones in extracellular electron transfer. Nature 405: 94-97.

(15) Poch M, Bezkorovainy A. 1991. Bovine milk $\kappa$-casein trypsin digest is a growth enhancer for the genus Bifidobacterium. J Agric Food Chem 39: 73-77.

(16) Rasic JL, Kurmsnn JA. 1983. Effect of substrate on the growth of bifidobacteria. In Bifidobacteria and Their Role, Birkh User Verlag, Basel, Switzerland, p. 42.

(17) Satomi K, Kurihara H, Isawa K, Mori H, Kaneko T. 1999. Effects of culture-powder of Propionibacterium freudenreichii ET-3 on fecal microflora of normal adults. Bioscience Microflora 18: 27-30.

(18) Zuccato E, Venturi M, Leo GD, Colombo L, Bertolo C, Doldi SB, Mussini E. 1993. Role of bile acids and metabolitic activity of colonic bacteria after cholecystectomy. Dig Dis Sci 38: 514-519. 